center of the odontoblast layer between the dentin cells can be elearly recognized. They pass from the parietal sheath or the nerves of the dentin germ and can be followed through the whole odontoblast layer beyond the boundaries of the dentin.

In Figure 19 the entrance of nerves into the dentin can be seen still more clearly. One of the axis cylinders is seen to terminate in a knob-like ending in the dentin, and another one terminate in a similar way before reaching the dentin, i. e., in the odontoblast layer.

The course of the nerves in the odontoblast layer varies greatly. They often traverse the layer in the same direction as the dentin channels and the odontoblasts. In some places they deviate from this direction. Sometimes they form a veritable plexus with all their small lateral branches. The nerve fibrils apparently originate from cells and cell nuclei. If one looks carefully it will be seen, however, that they are merely close to the cells and really pass alongside of them.

At the point of division of the nerves of the odontoblast layer I have frequently seen one or more spindleshaped enlargements. Such places are shown in Figure 20.

In conclusion, I take the liberty of demonstrating in Figure 21 the course of the nerve fibers within the dentin channels in a cow's tooth. The preparation was made according to Golgi by the sublimate method after the specimen had previously been injected with methylene blue. The fibers of Tomes appear pale; the fibers that I call axis cylinders are darker and frequently form spirals around the dentin fibers. They are situated between the walls of the dentin channels and the fibers of Tomes.

Basing on a series of histologic facts that I have attempted to illustrate to you with the aid of a few photographic slides of some of my specimens, I believe I have demonstrated the generally accepted odontoblast theory, the theory of dentin formation and of the sensibility of the dentin are untenable. While I do not dare to substitute a new and complete theory for the old one, I hope, nevertheless, to have contributed a few new facts by my investigations that may form the basis of an odontoblast theory that will satisfy us both in an anatomic and physiologic sense.

\section{DIABETES AND OBSCURE PHYSIOLOGY.}

CARL RAMUS, M.D.

Assistant Surgeon in the United States Public Health and Marine Hospital Service.

SAN FRANCISCO.

Of the many unsolved problems in pathology none perhaps possesses a greater interest to both theorist and practitioner than does the or:gin of diabetes mellitus. Its etiology is even yet shrouded in mystery, notwithstanding the enormous amount of study that has been devoted to it, and the situation may be summarized in the statement that of no other pathologic condition is there so much known, and yet so little. Many and various have been the theories advanced from time to time, cach having a few facts apparently supporting it, but as they conflicted with each other in important particulars, one after another they were abandoned, for the advancement in physiologic chemistry made it possible more and more to explain the different and complex processes by means of which the several classes of foods passed through stage after stage of preparation and claboration until they were fit to become incorporated into the tissues of highly organized beings. One after another the more conspicuous organs were taxed with the responsibility, and were believed to be the seat of the diabetic phenomena. But with the progress of seience the theories on which these opinions had been based were discredited, and they were reluctantly given up.

The theory that will be set forth in this paper is believed to be new as concerns its application to diabetes mellitus; but it has, however, appeared previously in medical literature in relation to purely physiologic researches concerning the ultimate state of the carbohydrate foodstuffs immediately preceding their absorption into the tissues. The various known facts on which this hypothesis is erected will be cited as required, not always chronologically, but in the order in which they apply here.

The inquiry concerns physiology as well as pathology, and, if the theory is correct, is as important to one as to the other. But before making any definite statements it will be in order to review briefly the experimental and clinical knowledge of diabetes mellitus, adding thereto our own observations.

The history of the scientific study of the subject up to the present, may with propriety be divided into four periods.

The first was inaugurated by the brilliant "piqúere" experiments of Claude Bernard. The important results finally arrived at through this class of experiments were, first, that violent stimulation applied to various parts of the nervous system was generally followed by glycosuria; second, that this was the result of a sudden liberation of glycogen in excess sufficient to pass through the renal parenchyma. Here we have strong evidence to show that under some conditions the nervous elements alone may be concerned in the production of some of the diabetic phenomena. This possibility has been further strengthened, in some instances, by necropsies showing definite pathologic conditions in important nervous locations. To this class may perhaps belong the occasional instances of diabetes following severe shocks of emotional and psychic nature; also those grave cases where no morbid anatomy could be found.

The second period began with the series of experiments that demonstrated the power of certain drugs, notably phloridzin, to produce temporary glycosuria. But the glycosuria obtained from phloridzin was found to be very different from that derived from the preceding experiments on the nervous systems. According to Von Mering and others it does not appear to depend upon glycogen liberation, the blood being poor in dextrose, and the glycosuria occurring in starving animals, and even after extirpation of the liver. The dextrose here must be formed principally from the albuminous tissues, and it seems probable that the glycosuria is the result of an altered condition of the renal functional tissue, brought about by direct action of the poison itself. This opinion is held by no less an authority than Von Noorden, who suggests the possibility that in some clinical cases the glycosuria may be the result of unknown toxins analogous to phloridzin circulating in the blood and acting on the kidneys. It seems evident from these researches that there are poisons existing in Nature capable of causing diabetic symptoms through their influence on the renal functions, and therefore clinical examples of diabetes mellitus from such sources are not at all beyond the range of possibility. There is no question but that the kidneys frequently exhibit pathologic conditions in diabetes, many showing catarrhal nephritis and at times fatty infiltration. One authority 
states that albuminuria is present in about one-third of all cases. Of course much of such pathology is without doubt sccondary, being the result of long overwork in excreting water, and irritation from the constant transit of sugar. However that may be, the results of the phloridzin tests must be considered to occupy an important place in the literature of this disease.

The third, or pancreatic, period marks the most important advance thus far made in the study of diabetes mellitus, bringing also new discoveries in physiology. Since attention began to be seriously directed to the pancreas as the organ most concerned, surprising and farreaching facts have been brought to light. The pathologic findings in about 50 per cent. of the necropsies showed plainly that the functions of this gland were interfered with or suppressed, and these observations perhaps inspired the painstaking and conclusive experiments of Von Mering and Minkowski, and also, independently, of di Dominicis, an Italian scientist. The importance of the work of these men in this field is simply incalculable. It has demonstrated beyond all reasonable doubt that removal or total suppression of function of the pancreas is invariably followed by typical diabetes mellitus. Since such experiments in the human species are entirely out of the question, it follows that these scientists were compelled to obtain their results from careful tests made on the lower animals, dogs having been selected for the purpose. The objection might be-and has been-urged that while the results of such experiments were probably conclusive in regard to the lower animals, they would not necessarily hold good for mankind. In reply to this it is now possible to cite at least one case in man which may be considered as experimental in the diabetic sense. It is referred to by Osler as a patient of W. T. Bull who died of diabetes after extirpation of the pancreas. The previously referred to investigators further showed that the antidiabetic property does not reside in the pancreatic juice; that a small portion of the pancreas, with its blood supply, remaining in the body prevents diabetes; that practically total extirpation of the organ is necessary in order to produce diabetes. They concluded that the action of the pancreas on the excretion of sugar is probably specific, no other organ possessing it. The latter statement is perhaps too radical in view of more recent studies of the suprarenal capsules. This paper will, it is believed, furnish evidence that will further strengthen the belief in the pancreatic origin of diabetes mellitus, possibly in all of the true cases; but no effort will be made to prove that all cases of apparent diabetes mellitus are necessarily pancreatic in origin, or can be explained by any one theory. But to return. The most significant facts shown in the pancreatic experiments were, that the pancreatic juice had no influence on the production of diabetes, and that the presence of a small portion of the pancreas prevented its occurrence. Of course the only explanation is that the pancreas acts in a manner similar to the ductless glands and that its internal secretion is of far more importance than its digestive contribution.

After consideration of the results derived from pancreatic sources it seems unreasonable to doubt that many cases of diabetes, probably the majority, have their origin in diseases of the pancreas. Lépine's experiments and theory furnish more evidence in support of the conclusions of Von Mering and Minkowski, and di Dominicis, but as it forms a strong link in the chain of evidence favoring the theory to be put forward here, its consideration will be postponed until it is taken up in proper order.
The fourth period in diabetic research may be said to have begun with Crofton's discovery of the relation thereto of the suprarenal capsules, made, I think, in 1901, The present writer regrets not having been enabled to more than glance at the important and very interesting work recently done as a result of this discovery. But the most pertinent fact demonstrated seems to have bien that injection of adrenal extracts can cause glycosuria. Previous workers have shown that several chemiical substances are able to induce glycosuria, but none of these have been shown, as yet, to occur naturally in human or animal bodies.

At last, however, a substance physiologically present is found to have the property of causing glycosuria when administered under certain conditions. A wide range of nossibility is thus unrolled, and a new field, nearly unexplored, presents itself for investigation. Much testimony has since accumulated, confirming the first important discovery and bringing to light new facts, several of which must be referred to.

It was stated in one publication that adrenal extract had been found to have the additional property of converting starch into dextrose. Herter, in 1902, found that when a solution of adrenalin chlorid was applied to the surface of a dog's pancreas glycosuria resulted. He also found, however, that several foreign substances, known to possess power as reducing agents, were able to effect the same, while oxidizing agents had no effect whatever. Herter concluded from these experiments that adrenal secretion produced glycosuria by means of its reducing power of the pancreatic tissue. I shall have occasion to refer again to this subject.

Having enumerated the main conditions now known to bear a causative relation to diabetes mellitus, or to glycosuria, the physiologic aspect of the matter must receive attention. For the purposes of this paper, the carbohydrate group of foodstuffs is the one mainly to be considered. Of course we are aware that in severe glycosuria dextrose may be formed from albuminous tissues, and even from fat to a slight extent, but the most important sources of dextrose are admitted by all to be the other carbohydrates. Physiologic chemistry has hitherto been unable to follow the carbohydrates beyond the dextrose stage, and there has consequently been a gap in our knowledge between the stage and the final assimilation by the tissues; or rather, the consensus of opinion seems to be that normal tissues absorb dextrose as dextrose, without further transformation. From this idea a theory to account for diabetic phenomena was formerly advanced postulating disturbance in the tissues of the function to absorb dextrose. But there is evidence on record strongly implying that dextrose does not express the final effort of Nature in the preparation of the carbohydrates for nutritive purposes.

In 1892 I épine found that fresh normal blood, after being kept in an incubator at physiologic temperature for some time, contained decidedly less dextrose than at the beginning of the experiment. In diabetic blood under the same conditions the loss in dextrose was not so pronounced. While not as yet confirmed, that I am aware of, by later workers, this demonstration not only receives confirmation from some of much earlier date, but is thereby actually much extended. Lépine's work in this direction seems to have proceeded no farther than the observation that dextrose was lessened in amount in blood which had stood some time at normal temperature. It stands to reason that if dextrose is diminished under such conditions something else must be formed. The next thing, then, to determine is, if 
possible, what this something is, and therefore another series of experiments will now have to be referred to.

In $18 \% 2$ Ford made a number of careful tests and elaborate calculations in relation to postmortem chemical changes occurring in normal blood and tissues. $\mathrm{He}$ worked on the theory that sugar is first converted into alcohol before it is possible for the tissues to derive benefit from it. In a large number of experiments a substance resembling alcohol, physically and chemically, was invariably recovered in minute amounts from fresh blood and tissue; from putrescent blood and tissue a much greater amount of this substance was always obtained. The amount obtained by experiment corresponded approximately to the amount of alcohol calculated, based on the amount of carbon ingested and on that exhaled. The smallest amount of alcohol was obtained from fresh liver tissue, and by far the largest from putrescent liver tissue. It is hardly conceivable that this alcohol, if such it was, resulted from anything else than the dextrose in the circulation, and in the case of the putrescent liver tissue from the store of glycogen previously liberated as dextrose. 'The tests on which Ford relied to demonstrate that the substance he obtained was alcohol were the us'ial ones then known to chemistry.

Ford's remarkable researches received corroboration in tl?o main idea in 1873, when Dupré and Béchamp-it would appear independently-discovered a substance resembling alcohol in normal urine, and from persons who had not exhibited alcohol for a long time. This discovery is also said to have been first made by Lieben in 1870 , and he also observed the phenomenon in the urine of dogs, horses and lions. In 1875 Rejewski obtained the substance from the urine of rabbits.

The statement that the substance found by Ford and the others was really alcohol, has been contested, as might naturally have been expected. The substance in question responded to the chromic acid test and the iodoform test; its watery solution had a lighter specific gravity than water; it yielded acetic acid on oxidation; and concentrated solutions were inflammable; the optical appearance in the conducting tubes, as noticed by Ford, resembled that of alcohol. Commenting on the fact that this physiologic substance reacts to all the known tests for alcohol, H. C. Wood very pertinently observes that "possessing the physical and chemical characters of alcohol, to ordinary minds it is alcohol."

The experimental testimony is conflicting as to the exact place of alcohol as a nutritive agent, its relation to heat production, electro-chemical action, carbonic acid and urea elimination, etc. But there is no question of the fact that alcohol is burnt up, or loses its identity, in the system, and that practically none of that ingested is eliminated as alcohol, unless administered in toxic amounts. It must be capable, therefore, of supplying, to an extent, the needs of the organism in health, and far more so in some pathologic conditions-a matter of every-day clinical experience.

In the light of present knowledge it would appear probable that the asserted conversion of dextrose into alcohol in the system is effected through the agency of an enzyme normally present therein. Our own experiments along this line, made in 1901-1902, are now in order. I am under obligations to Surgeon P. M. Carrington, in command at Fort Stanton, N. M., for extending to me such privileges as the laboratory of that station afforded while engaged in this work.

Solutions of dextrose, sterilized by boiling, were treated when cool with pancreatin preparations, and then set by in a warm place. After a number of hours small portions of the mixtures were distilled off and subjected to the iodoform and chromic acid tests for alcohol, giving in each case a positive reaction. More solutions were prepared and analyzed quantitatively, before and after prolonged treatment with pancreatin, to determine if any variation occurred in the percentage of sugar. A large number of these tests demonstrated a slight loss of sugar in nearly every case after having been acted on for some time by the pancreatin. Solutions of levulose tested before and after in the same manner showed a greater proportional loss of sugar than did those of dextrose. It seems hardly necessary to add that in each experiment the amount of water lost by evaporation during the period of fermentation was replaced by distilled water before reanalysis; and that the differing affinities of dextrose and of levulose for copper hydroxid were taken into account. Solutions of cane sugar treated with pancreatin gave, in their distillates, the iodoform and the chromic acid reactions for alcohol. The vapor from a dextrose and pancreatin soIution imparted a slight cloudiness to lime water, but as the experiment was rather carelessly made, and, unfortunately, was not repeated, it can not be considered as positive. Pancreatin itself contains considerable soluble material capable of reducing copper solutions, and this self-contained reducing agent undoubtedly took some part in the fermentative processes. Being apparently for the most part dextrose its presence should not detract from the importance of the other findings, except in the instance where an alcoholic distillate was obtained from a cane sugar and pancreatin solution. A strong point against any undue importance of this factor in the other experiments is that dextrose and levulose solutions did not lose in sugar proportionally, and that the variation in the levulose was inversely what might have been expected from ordinary accidental fermentation.

Assuming, then, the correctness in the main of all the experimental findings herein adduced, the situation physiologically would appear thus:

1. That in normal blood dextrose undergoes chemical modification before absorption by the tissues.

2. That in normal blood alcohol develops from disintegration of dextrose.

3. That alcohol is present in minute quantities in the urine of total abstainers.

4. That in chemically pure solutions of dextrose and levulose treated with pancreatin, sugar is lost and alcohol (and carbonic acid?) developed, the change being more marked in the case of levulose.

5. That the agent responsible for the chemical reaction is an enzyme normally present in blood.

6. That this enzyme has its origin in the pancreas alone, for when that organ is removed dextrose remains unchanged.

7. That interference with or disturbance of this function of the pancreas initiates a group of phenomena known clinically as diabetes mellitus.

\section{SOURCES OF ERROR.}

It is true that at the present time much of the experimental data referred to lacks corroboration by other observers, notably that of Lépine, Ford, and the present writer. I have not seen Ford's original paper, being indebted to the section on alcohol in H. C. Wood's Therapeutics for a synopsis of his results, and also much additional valuable information. But that there were sonrces of error in Ford's work seems probable when it 
is remembered that in his time bacteriology as a science hardly yet existed, and that therefore he may not have taken into account the action of saprophytic bacteria in the change from dextrose to alcohol. In this way it is likely that his results with putrescent tissues were partially erroneous. But in view of the very significant fact that he obtained traces of alcohol from fresh blood and tissue, the importance of any possible errors in connection with tissue having undergone bacterial action ought not to be exaggerated.

Such objections, however, can not be urged in regard to Lépine's results, for they were reached at a time when the principles of modern bacteriology were familiar to all contemporary scientists. Though Lépine is not on record, that I am aware of, as having demonstrated alcohol, he certainly has demonstrated the complementary fact, namely, the disappearance of dextrose in normal blood, and to a lesser extent in diabetic blood. Lépine's and Ford's separate blocks of evidence, when placed side by side, form a chemically perfect whole, the former having shown that sugar disappears, the latter that alcohol appears. Collateral testimony is afforded by the work of other investigators cited who demonstrated the presence of traces of alcohol in normal urine.

In our own work, which had for its object the demonstration of a pancreatic enzyme able to convert dextrose into alcohol, the principal source of error was infection of the solutions with saprophytic bacteria. By a process of exclusion it was made evident that the infection was derived from the commercial preparations of pancreatin, a fact of no importance whatever ordinarily, but of considerable in experiments of this nature. In only two instances were perfectly sterjle solutions made naturally. Efforts were then made to control bacterial action by means of small amounts of boric acid, oil of cloves, etc. It was found that in order to suppress saprophytic action much more of these substances was required than is currently believed, so much, in fact, that the action of the enzyme was nearly inhibited. By comparing the notes of a large number of experiments under these adverse conditions it was shown, however, that a slight loss of sugar nearly always resulted. The source of error here, if there was one, would have occurred in the manipulation, toward the end of each test, of the Fehling solution, by no means an ideal method where small differences have to be computed, but perhaps the best in the long run. The levulose preparations showed a greater loss of sugar than did those of dextrose. In solutions of either sugar with pancreatin where no antiseptic had been added, and where saprophytic action occurred, the loss of sugar in a given time was far greater, especially in the case of levulose. The infecting organism most in evidence was a large spore-forming chain-rod, generally alone, occasionally accompanied by other germs in small numbers. The yeast fungus was not found to be present at any time.

How much of the large amounts of sugars lost here, in excess of that lost where bacterial action was inhibited by antiseptics, depended upon the pancreatic enzyme, and how much was due to bacteria, we are unable at present to say, and can place reliance only on the results obtained where the action of bacteria was eliminated. It is certainly worthy of note, though, that the corresponding losses of dextrose and levulose under the same conditions of infection were so widely different as to imply something more at work than bacterial action alone.
Among the number of experiments made to demonstrate the development of alcohol were two in which, fortunately, no infection occurred and no antiseptic was employed. In these cases the principal source of error to be considered would have been in the chemical tests to which the distillates were subjected. Both the iodoform test and the chromic acid test are well known to react in the same manner with many substances besides ethyl alcohol. In this connection it was observed that the chromic acid test gave the same color reaction with methyl alcohol, dextrose, levulose, lactose and cane sugar. Seeing that both tests gave typical reactions with material derived from sources more likely in the natural order of things to generate ethyl alcohol than anything else; and seeing that such a result fits in very naturally with Lépine's and Ford's results; it must be admitted that strong reasons exist for presuming the reacting substance to have been alcohol.

An attempt will now be made to account for diabetic phenomena on the theory that they are the expression of an abnormal condition wherein the hypothetical alcoholic function of the pancreas is disturbed.

\section{DIABETES MELLITUS.}

If it is a fact that the normal internal secretion of the pancreas must convert dextrose into alcohol before the tissues can make use of it, then it naturally follows that a consideration of this obscure function would have importance in relation to true diabetes mellitus of pancreatic origin, as our knowledge stands at present. The various and diverse manifestations of the disease, when considered in this light, seem to fall easily into well-defined places, while at the same time theories placing the responsibility on other organs are seen to be untenable. These remarks are not intended to apply to other than pancreatic forms of diabetes, or glycosuria, for we believe that such may occur, and will venture a few remarks on them in their proper place. We will begin the discussion with the behavior of the liver in reference to pancreatic diabetes.

The most significant feature here is the absence of glycogen, practically, in the liver and muscular tissue. This, together with the notable excess of dextrose in the blood, suggested at least three theories, two implicating the liver; the first for over-activity, the second for under-activity; while the third placed the blame on the tissues in general as having partially lost their power to absorb dextrose. The pancreatic alcoholic theory at once clears up all this; the liver is seen to be merely doing its duty while the tissues, being unable to assimilate the unaltered dextrose furnished by the liver, are imperatively and without intermission demanding nourishment. But the nervous mechanism, which in health had apprised the liver of the needs of the tissues, is still uninjured, and the constant demand for food is as constantly responded to by the liver in the only way it is capable of doing; it has no time to store up glycogen, and the fact that the excess of dextrose given out is injurious to the organism implies no fault in the liver whatever, but actually the reverse. If the liver were the seat of the assumed normal alcoholic change in the condition of dextrose, then there would be found no free dextrose in the blood except in minute amounts like glycogen, for all sugar would be immediately seized and stored up as glycogen, never to be released except in the form of alcohol.

The existence of glycosuria and polyuria, without other evidences of nephritis, is merely symptomatic; the glycosuria because the normal percentage of sugar is ex- 
ceeded, and the polyuria because of the irritating action of dextrose on the renal tissue. Secondarily, of course, a true nephritis will probably occur if the condition is not ameliorated.

The remarkable and inexplicable property of levulose to form glycogen and diminish glycosuria can now be explained. Levulose is more rapidly and easily converted into alcohol than is dextrose by the pancreatic enzyme, and the reduced amount or power of the latter in diabetes is thus able to take care of considerably more levulose than dextrose, and the liver and muscular tissues are enabled to store a certain amount of glycogen.

As milk is comparatively well tolerated in diabetes, it follows that lactose must have properties somewhat similar to those of levulose. I have not experimented with lactose as with levulose, but would expect to find it somewhat similar in this respect. It is noteworthy that there is a curious analogy in the purely chemical and the purely physiologic contrasting properties of dextrose on the one hand, and levulose on the other. A definite amount of copper hydroxid will combine with more levulose and still more lactose than with dextrose; in physiology, on our theory, levulose and lactose are more readily appropriated by the system than is dextrose, the fact being especially noticeable in diabetes mellitus.

The increased elimination of urea and uric acid is natural and proper, since in this disease a larger amount of albuminous material is employed, irrespective of the kind of food ingested, for if that is lacking in proteids the deficit is made up from the tissues themselves.

The fatty condition of the blood is apparently a further effort of Nature to store glycogen and form dextrose. It has been shown that fat in extreme conditions, as in starvation, can form glycogen, and therefore indirectly, dextrose. Diabetes mellitus is merely another aspect of starvation. The greater part of the large amount of food ingested is useless to the organism, for the system is lacking in the element whose presence alone can render it available for its needs. The condition has its analogy in the fable of Tantalus, striving forever to sip "The cup that flies from his arid lip."

The tendency toward acidity of the blood in later stages of the disease, with the development of acetone, diacetic acid and beta-oxybutyric acid, may be referred to several causes. The alkalinity of the intestinal tract depends principally on the bile and pancreatic juice. The liver being constantly overtaxed in the exercise of the glycogenic function and the preparation of proteids and elimination of urea, must in time neglect the biliary function as being subordinate to the ethers in importance; while the pancreas, diseased in one of its most important functions, can hardly be expected to keep up the standard of its intestinal secretion. It follows consequently that the intestinal juices become in time deteriorated, thus favoring the development of acetic and lactic and other acid substances. These eventually begin to enter the portal circulation, and, after a time, the general circulation, and ultimately change the reaction of the blood. The normal power of the liver to arrest and render harmless the poisonous substances developed in the intestines, becomes diminished, and these adding their baneful influences to that of the general hyperacidity, finally give rise to comatose symptoms.

\section{OTHER FORMS OF DIABETES. RENAL GLYCOSURIA.}

There is evidence showing the possibility of diabetes in some cases arising from the kidneys themselves. In the first place, albuminuria and different degrees of nephritis have been observed in perhaps one-third of all cases of diabetes, while definite pancreatic lesions have hardly occurred in more than 50 per cent. of cases.

While in many of the cases exhibiting renal involvement the pathology may be merely secondary to pancreatic disease, it can not always be proven that such is the case. In the series of experiments of which the one employing phloridzin is the type, we are shown that renal diabetes is a possibility, either as resulting from disturbance of osmotic equilibrium, or it may be, of the renal synthetic functions. The fact that severe forms of Bright's disease do not often show glycosuria, complicates the inquiry, and supports to some extent the theories relieving the kidneys of responsibility in glycosuria. The question of renal involvement will be again alluded to under the head of therapeutic suggestions.

\section{GLYCOSURIA OF NERVOUS ORIGIN.}

That pathologic conditions involving the nervous system can induce glycosuria has been shown cxperimentally and clinically; but even here the glycosuria must be indirectly referred to the agency of one or more of the internal organs whose ordinary routine has been disturbed by disturbance of its innervation. The osmotic balance of the kidneys may be disordered; the glycogenic function of the liver, the alcoholic function of the pancreas, or the internal secretion of the adrenals - perhaps also that of the thyroid-may be altered. Thus it may be inferred that defective innervation can, theoretically, inaugurate glycosuria through a number of sources.

\section{ADRENAI DIABETES.}

With the discovery of the power of adrenal extracts to cause glycosuria (and to convert starch into dextrose?) a new and interesting element was added to the subject of diabetes. By applying these facts to the present inquiry, together with the known physiologic action of the glandular extract, and assuming the existence of a condition of the adrenals causing over-production of their internal secretion, it would appear that glycosuria may be induced in four different ways, or by a combination of one or more of them, namely, by increased blood pressure; by irritation of the renal parenchyma; by inhibition of the alcoholic function of the pancreas; and by direct action on the stored up glycogen, converting it into dextrose.

In the event of under-production of the adrenal secretion we might assume that one of the first compensatory efforts of Nature would be stimulation of the alcoholic function of the pancreas, the object being to make up the deficit in adrenal secretion by an increase in the production of alcohol, for the physiologic action of the latter ought to enable it to partially substitute the former temporarily. Such a possible substitution has its analogy in the splitting up of the proteid molecule with the formation of an alkaline ammonium compound when from any cause there is a tendency toward acidity of the blood which can not be controlled by the alkaline substances normally present. But since over-stimulation of any organ tends eventually to loss of function, it would follow, on this theory, that a time would come when the pancreas could no longer exercise its alcoholic function sufficiently for the needs of the system. Then the liver would endeavor to supply the want by liberating an increased amount of dextrose, and it would then be only a question of time when glycosuria and the concomitant symptoms of pancreatic diabetes appeared. It may be reasoned in this way that the pancreatic and 
adrenal functions are intimately related; the adrenal secretion may be essential in regulating the exercise of the pancreatic alcoholic function in health, while in the event of insufficiency of the former the latter would be stimulated.

\section{EXPERIMENTAL CONFIRMATION.}

It is much to be regretted that confirmation by other observers is yet lacking of much of the data essential to the theory herein advanced. Even Lépine's results, significant as they are, do not seem to have received corroboration; while Ford's work is never alluded to in treatises on diabetes and seems to have escaped attention altogether. My own work is very incomplete at best, but as far as it goes I feel that it can not be altogether unimportant, and trust that it will soon be taken up by others, along with Lépine's and Ford's observations. I intended to submit my results for publication early in 1902, as soon as the series of experiments outlined should have been finished. But unforeseen interruptions occurred and official duties in connection with the routine work of the Sanatorium interfered; and as there is even yet no immediate prospect of my being able to resume the experiments, I have decided to submit such as have been made, together with the theory they suggest, in the hope that the work may be investigated and extended by others, and its value, if it possesses any, be determined.

Bccause of the presence of saprophytes in the commercial dried preparations of pancreatin, I would suggest that they be discarded in experiments of this nature, and that the fresh organs of animals recently killed be employed. The amount of dextrose or reducing substance in the gland itself or its extract should be determined before treating the sugar solutions. Sufficient alcoholic liquid should be obtained so that its physical and chemical characters can be established with certainty. In the chemical equilibrium of the system the amount of alcohol present must be very small at any time compared to that of dextrose, and therefore in any experimental solution the relative proportions may be expected to approximate similarly; for the principle of "reversibility of enzymes" should be in operation here as elsewhere in the system. If alcohol is really formed the amount should of course bear definite relation to the amount of sugar lost. The fact that small quantities of alcohol are produced in the intestines should not be ignored even though it can not have great significance here, since alcoholic development occurred under conditions excluding intestinal co-operation. The reactions with levulose and lactose should be particularly noted because of their peculiar properties in diabetes mellitus. In all cases control tests would be imperative. The action of adrenal extracts on sugars and starches ought also to receive further attention. The fact should be taken into account that the pancreatic alcoholic enzyme must be present, not only in the pancreas, but throughout the system to a certain extent. It has been asserted that glycerin may assist in the formation of glycogen; it would also be worth while to investigate the behavior of ingested alcohol in regard to that function, remembering that Ford found less free alcohol in fresh liver than in any other tissue. In testing diabetic material for alcohol one would expect to encounter much less than in the normal, perhaps none at all. The chromic acid and iodoform tests would be valueless here because of the likelihood of the presence of acetone, a substance giving the same reactions as alcohol with these tests.
THERAPEUTIC SUGGESTIONS.

If the theory set forth here is the correct one, then the importance of alcohol in the treatment of diabetes is the most obvious feature that presents itself. Based on clinical observations, the best authorities are already nearly agreed as to the beneficial influence exerted by alcohol in diabetes when given in moderation. Clearly on our hypothesis reasonable amounts of alcohol will relieve the pancreas, liver and kidneys for the time being by furnishing ready prepared nourishment and force to the starving tissues; there will be temporary suspension of the immense and constant and all but unavailing efforts of those organs to relieve the strained situation. Very possibly some storage of glycogen may be accomplished during the period of relief thus afforded.

With regard to the administration of pancreatic extracts, Von Noorden and others have not had very encouraging results. Only two methods would appear available in the employment of these preparations. One way would be to give them with or immediately preceding meals, the idea being to take advantage of the presence of the alkaline saliva, since pancreatin can not act in acid media; the doses should be large in order to assure the absorption of an amount of the enzyme, for the major portion would soon be destroyed in the stomach. The other way would be incorporation in alkaline nutrient enemata and injection into the rectun or colon.

I have seen great apparent benefit follow the use of mescal de tequila in two cases, both of them young subjects. Whether this was merely from the alcohol present, or because of specific properties possessed by the drink, I am unable to say, though inclined to put some faith in the latter possibility.

Mescal is a fermented intoxicating liquor derived from the maguey plant, native to southern New Mexico, Arizona and Old Mexico. The unfermented juice of the plant is called pulque, and it, as well as mescal, has a great reputation among the natives as a specific for all "kidney" diseases, pulque being the most thought of. With them diabetes is naturally considered a disease of the kidneys, but there really appears to be some firm ground on which the popular faith in the drug rests, both as regards diabetes and Bright's disease. That it exerts some special influence on the kidneys is certain. In several cases of Bright's disease exp rimented on at Fort Stanton, N. M.. it was found that ehronic interstitial cases were much benefited in a short time, both as regarded the amounts of urine and albumin. Chronic parenchymatous cases were also speedily brought under the influence of the remedy, showing lessening of albumin, but there was at the same time a tendency toward suppression which had with it an element of danger. The cerebral effects of overdoses were unpleasant and were induced by much smaller amounts than mere whisky would have done.

In this connection I have to acknowledge my indebtedness to Dr. H. K. Pangburn, Acting Assistant Surgeon, and to Mr. J. E. Beck, Senior Pharmacist, U. S. MI. H. $\mathrm{S}$.; the former for daily quantitative urinalyses in the cases under observation; the latter for pharmaceutic preparations of extract of the maguey plant.

In view of its effects in Bright's disease, and remembering the possibility of renal diabetes, the suggestion may be hazarded that while the specific action of the maguey plant on the kidneys would perhaps account for its beneficial action in cases of diabetes of 
renal origin, it might not possess any value in the true pancreatic variety.

\section{ETIIICAL CONSIDERATIONS.}

The assumption of the existence of the alcoholic function of the pancreas, on account of its relation to nutrition in general, diet, habits, and the treatment of diabetes, brings up a final and important consideration, namely, the question of intemperance.

It has been suggested that were a theory such as this one to become generally accepted it would probably exert a baneful influence on the race in general by furnishing a physiologic excuse for increasing the consumption of alcoholic beverages.

When looked at superficially such a fear might seem reasonable; but when examined more critically it is seen to have no foundation.

Everyone is aware that perfect physical health means nothing more or less than perfect functional activity as established by Nature's immutable laws. All efforts made by man to modify these functions have signally failed, and no matter what abnormal extent of tolerance may have been achieved by the pleasure seeker, the epicure or the heavy drinker, their reckoning with Nature must come at last, for they have reckoned without their host.

The man would be a poor physician indeed who saw fit to recommend to his patients the consumption of unusual amounts of cheap glucose candy in place of a portion of their ordinary diet, because he had learned in the study of physiology that glucose represented an advanced effort of Nature in the preparation of the carbohydrate food group.

Exactly the same may be said in the case of alcohol and of the medical man who would advise the employment in health of alcohol as a substitute for a certain amount of food, because alcohol appears to be the final product of an important section of the foods, and, by such specious reasoning, more easy and natural to assimilate than ordinary foods. Nature has made abundant provision and has combined the various nutritive principles in a manner that can not be improved on in the essential particulars. No matter what the advance made in our knowledge of physiologic chemistry, mankind still have to depend on Nature's unvarying processes for their continued existence, and, physiologically, are neither better nor worse than they were ages ago. The natural processes involved in digestion, nutrition and assimilation are not intended to be altered in any way, either by increasing or lessening their scope. The fact that foodstuffs occur in forms that require such extensive elaboration in the system is sufficient evidence of the wisdom of such an arrangement.

When considered in this light it will be seen that the alcoholic theory in physiology is in reality a strong argument in favor of temperance. In health man needs no ready prepared alcohol whatever, except such as occurs naturally in some of the foods in small quantities.

But the situation is very different indeed when the management of disease has to be confronted. If alcohol is a physiologic necessity, as produced in the body in health, then it follows that in the treatment of many diseases its employment as a medicine would be of the greatest possible advantage to the patient. The most obvious indications for its administration would be in those pathologic conditions wherein the mysterious protective agencies are summoned to exercise their functions to the utmost limit in order to cope successfully with some foreign invasion or auto-intoxication which menaces the integrity of the system. Nearly every medical man of experience will concur in the opinion just expressed; while as regards the few who see fit to exclude alcohol altogether, on the ground of religious or other principles, no amount of argument, laboratory experiment or clinical experience, however convincing, can be expected to have weight.

In conclusion, then, from the standpoint of the alcoholic theory in physiology, based upon experimenta] and clinical results, it would appear that in alcohol we possess the most efficient agent for sustaining the vital forces of the system when its functional activities are paralyzed by the inroads of various diseases, whose presence necessitates the subordination of all other functions to those immediately engaged in the work of repelling the invasion.

\section{RADIUM AND RADIANT ENERGY.* \\ JOHN INGLIS, A.M., M.D. \\ Member Colorado State Board of Medical Examiners. PUEBLO, COLO.}

The discovery of radium is of universal interest. There is scarcely a science that has not been affected by it. But it can be of no greater interest to any one than to physicians, because of its therapeutic possibilities. The termination "um" indicates that radium has been added to the family of elements.

A substance to be admitted into the list of elements must have the following qualification: It must have a spark spectrum of its own. This spark spectrum must be measured and be different from all other forms of known matter. Radium fulfills this requirement. Demarcay has shown that radium has a spark spectrum of fifteon lines, with no lines belonging to any other element. The discovery of radium during the closing vears of the nineteenth century was one of the most wonderful achievements of that century, from a scientific point of view. In some ways it amounts almost to a revolution in our theory of matter. The study of physics and chemistry has made the most rap:d progress of any of the sciences in the past few years. The textbooks which the youngest of us used are out of date, so rapid have been the changes and progress along this line of study.

So it may be well to note, in introducing this subject, some of the more essential changes, without understanding which we can not gain a clear idea of the subject before us.

We were taught as school boys that that smallest particle of hydrogen, called an atom, was the least mass of matter known. Since that time, however, Thomson has discovered that all space is filled with inconceivable small corpuscles. These corpuseles, Professor Larkin tells us, are doubtless either electricity in its ultimate refinement or very closely allied to it. A Thomsonian corpuscle is only $1 / 1000$ the mass of an atom of hydrogen. They are so small that the wide spaces in a bar of steel or even in the diamond itself, permit these corpuscles to pass through them. They are positive or negative, electrically speaking. But the corpuscles flowing between all kinds of matter are negative.

Twenty-five hundred years ago Leucippus, the Greek philosopher, suggested the atomic theory of matter. Dr. John Dalton, at the beginning of the nineteenth century, formulated it into the hypothes:s which we

* Read before the Pueblo County Medical Society. 